\title{
QUELLE DÉMARCHE D’AMÉNAGEMENT RETENIR \\ EN FORÊT TROPICALE HUMIDE ?
}

\section{J. VALEIX}

Couvrant un peu moins de 1,7 milliard d'hectares, soit la moitié de la surface forestière mondiale, les forêts tropicales, qu'elles soient situées en zone humide ou en zone sèche, sont essentiellement considérées, aux yeux de l'opinion publique internationale, pour la très grande diversité biologique qu'elles abritent, voire pour leur contribution jugée positive au cycle de l'eau et, plus récemment, à celui du carbone.

Toutefois, il est peu fait état du demi-milliard d'habitants qui vivent en zone boisée tropicale et dont le niveau de vie dépend parfois étroitement des divers produits qu'ils prélèvent en forêt en vue de leur utilisation directe ou de leur commercialisation. II faut certainement en rechercher la raison dans les craintes qu'inspire le rythme de la déforestation dans les pays tropicaux et qui ont été récemment ravivées par les incendies spectaculaires de formations forestières survenus en Indonésie en 1997 puis au Brésil en 1998, sans oublier les reportages stigmatisant les effets négatifs d'une exploitation jugée excessive des ressources forestières. Mais, peut-on raisonnablement oublier que les forêts tropicales sont essentiellement localisées dans des pays où la sécurité alimentaire, la lutte contre la pauvreté et, partant, le développement économique constituent des enjeux majeurs ?

Dans de tels contextes, la valorisation économique des forêts tropicales, notamment par l'intermédiaire de modes de gestion viables à terme, constitue un objectif majeur qui se doit d'intégrer, dans une approche globale, le rôle multifonctionnel de ces forêts ainsi que la diversité des usages qui y sont pratiqués.

Encore faut-il que la démarche complexe et exigeante de l'aménagement forestier, traitement spécifique d'une partie du territoire, d'une part, véritable processus de négociation et de décision, d'autre part, ne soit pas réduite à ses seuls itinéraires techniques et à une approche par trop sylvicole. En effet, au cours du vingtième siècle, certains forestiers occidentaux ont cherché à transposer aux forêts tropicales les techniques et les outils mis au point dans la gestion des forêts tempérées. Or, malgré le nombre et la diversité des expériences réalisées en Asie, en Afrique et en Amérique latine par des forestiers de différentes cultures, force est de constater que la très grande majorité des itinéraires techniques, certes jugés pertinents à l'échelle expérimentale, n'ont finalement pas pu être généralisés, soit par manque de moyens humains et financiers, soit parce que les motivations profondes des acteurs locaux étaient d'une toute autre nature, soit encore parce que les contextes institutionnels et législatifs des pays concernés n'y étaient pas favorables. 


\section{J. VALEIX}

Aussi, alors que les notions d'aménagement forestier et de gestion multifonctionnelle et intégrée des forêts progressent significativement dans les débats internationaux, régionaux et nationaux, il peut s'avérer profitable, à la lumière des expériences passées, de revisiter certains fondements de notre pensée forestière et de s'interroger sur les facteurs conditionnant l'aménagement et la gestion réellement intégrés des forêts tropicales.

\section{LES PRINCIPAUX OBSTACLES AUX DÉMARCHES INTÉGRÉES D'AMÉNAGEMENT DES FORÊTS TROPICALES}

\section{L'impact des dynamiques démographiques et foncières sur les forêts tropicales}

Contrairement aux pays d'Europe occidentale où le foncier forestier et le foncier agricole ont pu être relativement stabilisés au cours du XIXe siècle avec, dès lors, une inversion de tendance conduisant, ces cent cinquante dernières années, à une augmentation substantielle des taux de boisement, les dynamiques d'affectation et d'occupation des terres dans les pays du Sud sont d'une toute autre nature. Elles s'exercent en effet sous l'action combinée de fortes croissances démographiques, de politiques volontaristes d'aménagement voire de colonisation des territoires, enfin de l'impact sur les forêts des options prises en matière de développement national et local.

Ainsi, sur une superficie des forêts tropicales estimée, au niveau mondial, à un peu moins de 1,7 milliard d'hectares, ce sont, selon la FAO, entre 10 et 15 millions d'hectares de forêts tropicales qui sont déforestés annuellement, ce qui correspond à un taux de déforestation légèrement inférieur à $1 \%$ par an. De tels chiffres, sans cesse repris dans les rapports internationaux sur les forêts tropicales, présentent certes l'avantage d'une vision globale et moyenne. Ils ne doivent toutefois pas masquer les très grandes hétérogénéités qui existent en ce domaine entre les différents pays tropicaux. Quelle commune mesure en effet entre les dynamiques non maîtrisées des fronts pionniers en œuvre actuellement au Cameroun, au détriment très net des forêts tropicales de ce pays, et celles, symboliques, du Gabon, pays faiblement peuplé puisqu'il ne compte qu'un million d'habitants et qu'un tiers de la population est passé du milieu rural au milieu urbain en l'espace d'une génération?

De même, les dynamiques des fronts pionniers ne doivent pas être uniquement analysées spatialement à un instant donné. Pour bien les comprendre, il est nécessaire de les inscrire dans le temps. En Indonésie, par exemple, les densités extrêmes de population observées dans l'île principale de Java, voire dans celles de Madura et de Bali, de l'ordre du millier d'habitants au $\mathrm{km}^{2}$, sont à l'origine d'une politique nationale à long terme et volontariste de migration organisée d'une partie de ces populations rurales vers des îles considérées comme "périphériques" et, en tout état de cause, nettement moins peuplées. Ce fut le cas dans un premier temps de Sumatra, où les densités de population sont comprises, d'une province à l'autre, encore actuellement, entre 20 et 40 habitants au $\mathrm{km}^{2}$, mais aussi des îles Célèbes. C'est au tour actuellement de Kalimantan, où les densités sont inférieures à 5 habitants au $\mathrm{km}^{2}$, d'accueillir les migrants originaires de Java. Cette politique, dénommée "Transmigraci", couplée par ailleurs avec de grandes opérations industrielles de plantations, qu'elles soient agricoles ou forestières, s'est bien évidemment traduite par des déforestations de très grande ampleur, les spectaculaires feux de forêts survenus en Indonésie en 1997 n'en étant que la manifestation la plus visible aux yeux de l'opinion publique internationale.

Sur un autre continent, la dynamique de déforestation observée en Amazonie correspond également à une politique volontariste de colonisation spatiale et s'appuie sur des migrations internes de grande importance, ce que résume le slogan brésilien : "des terres sans hommes pour des hommes sans terre". Là aussi, les feux de 1998 n'ont fait que révéler, suite aux conséquences climatiques locales des oscillations d'“El Niño", l'importance des phénomènes de déforestation en cours en Amazonie. 
Pour sa part, le passage, ces quarante dernières années, de la superficie forestière de Côte-d'Ivoire de 15 à environ 3 millions d'hectares est dû au choix d'un processus de développement et de diversification de l'économie agricole ivoirienne couplé avec l'immigration concomitante de populations soudaniennes, principalement d'origine burkinabée, fuyant la sécheresse et constituant de facto une main-d'œuvre essentielle pour permettre la mise en œuvre et la poursuite d'un tel processus de développement.

Comme le démontrent les dynamiques irréversibles de ces grands fronts pionniers, les politiques extra-sectorielles jouent clairement un rôle déterminant sur le devenir des forêts tropicales. Raisonner les meilleurs modes de gestion forestière durable ne peut donc en réalité avoir de sens que dans des contextes où le statut du foncier forestier est globalement maîtrisé et où le comportement spatio-temporel des populations locales peut être considéré comme stabilisé vis-à-vis de I'utilisation des espaces forestiers.

\section{Les risques engendrés par des surcapacités industrielles}

\section{- Le poids de l'Asie}

Dans un contexte de mondialisation croissante du marché international des bois tropicaux, l'Asie a clairement démontré en cette fin de siècle les risques que pouvait représenter, pour la viabilité des forêts tropicales, l'installation de surcapacités industrielles. Ainsi, focalisées par l'importance de la demande de la Chine, du Japon et de la Corée du Sud en bois semi-ouvrés et ouvrés, I'Indonésie et la Malaisie ont favorisé la mise en place sur leur territoire d'industries de transformation dont la capacité totale était très nettement supérieure aux capacités de production de leurs forêts tropicales. Elles ont pris également des mesures d'interdiction ou de très forte taxation des exportations de grumes, générant de facto une dévalorisation, par rapport au marché international, du marché local des grumes tropicales, d'une part, un manque relatif d'efficacité technico-économique de leurs entreprises de transformation, d'autre part.

De tels dysfonctionnements se sont inéluctablement traduits non seulement par des niveaux d'exploitation forestière extrêmement élevés mais aussi par de faibles taux de rendement de leurs procédés de transformation. En outre, les motivations de telles entreprises forestières, qui font généralement partie de larges conglomérats industriels et financiers, sont le plus souvent orientées par les exigences du court terme et par le caractère mobile des activités économiques et des capitaux financiers.

Les approvisionnements locaux ne suffisant donc plus, on vient d'assister au cours des années quatre-vingt-dix à la traduction, continent par continent, de la stratégie mondiale des firmes asiatiques, et ce par le biais de leur positionnement volontariste sur les deux zones d'approvisionnement majeures que constituent le bassin du Congo et l'Amazonie. Le risque d'une transposition à ces régions forestières des principaux dysfonctionnements observés jusqu'à présent en Asie du Sud-Est ne peut donc être écarté.

\section{- L'évolution risquée de l'Afrique tropicale humide}

Bien des pays africains de la zone tropicale humide, notamment dans le bassin du Congo, prônent actuellement l'industrialisation de leur filière-bois en vue de rechercher une meilleure valeur ajoutée de leur production et réglementent de manière de plus en plus volontariste l'exportation des grumes.

Au-delà du fait qu'une plus grande transformation sur place des produits forestiers n'est pas toujours synonyme d'un accroissement de la valeur ajoutée, nombreuses sont aujourd'hui les installations artisanales ou industrielles qui, bien que souhaitées par les dirigeants locaux, ont des taux de rendement extrêmement bas et gaspillent donc de manière visible le matériau bois. Certes, un 


\section{L'aménagement sans frontières}

sont bien connues des spécialistes en sciences humaines, bien des ONG environnementales ont régulièrement buté et butent d'ailleurs encore sur la place à réserver à l'homme, et plus particulièrement aux populations locales, dans les espaces naturels "tropicaux" considérés comme des hauts lieux de conservation de la diversité biologique. Ne voit-on pas encore de nos jours des projets internationaux de conservation de la nature envisager le déplacement de populations locales vivant dans tel espace "naturel" récemment érigé au rang d'aire protégée ? Une telle approche, par nature très simplificatrice et se basant sur une vision trop réductrice des rôles possibles des populations locales vis-à-vis de la conservation de la nature, est généralement condamnée à rencontrer de nombreuses difficultés sans négliger in fine les risques d'échec par rapport aux objectifs initialement fixés.

Rappelons à ce propos que, de nos jours, ce sont entre 400 et 500 millions d'habitants qui dépendent encore directement des divers produits tirés des forêts tropicales. En outre, il est de plus en plus admis qu'une politique crédible de conservation de la diversité biologique à l'échelle d'un pays ne peut s'en tenir à la constitution d'aires protégées qui ne représentent généralement que 5 à $15 \%$ de chaque territoire national. Elle se doit également de considérer le rôle et les contributions possibles des autres territoires dans ce domaine et chercher à définir une approche globale et cohérente des enjeux liés à la conservation et à l'utilisation de la diversité biologique.

\section{- Les limites de l'approche "productiviste"}

Faute d'une stabilité politique suffisante et d'une vision cherchant, d'une part, à pérenniser ses activités dans l'espace et dans le temps, d'autre part, à économiser les surfaces exploitées et à apporter la plus grande valeur ajoutée possible au matériau bois, l'exploitation tropicale s'est, jusqu'à présent et sur de nombreux continents, plutôt rapprochée d'une exploitation de type minier que de la gestion d'une ressource naturelle renouvelable, ceci malgré quelques inflexions récentes.

En effet, à défaut de pouvoir imaginer la faisabilité de rotations supérieures à cinquante ans, quelle autre signification que celle d'une exploitation minière peut-on accorder à l'exploitation intensive des forêts à Diptérocarpacées d'Asie du Sud-Est quand on sait que le prélèvement moyen d'une dizaine de pieds à l'hectare, pratique tout à fait courante dans cette région du monde, correspond, en cumulant l'extraction des bois hors de la forêt et la mortalité, immédiate ou différée, à un impact global généralement compris entre 100 et $200 \mathrm{~m}^{3}$ par hectare, soit un taux d'impact allant du quart à la moitié du capital ligneux initial sur pied.

De même la pratique, très souvent observée dans les pays du Bassin du Congo, des migrations dans l'espace et dans le temps des concessions forestières attribuées aux entreprises d'exploitation forestière ne s'explique-t-elle pas essentiellement par l'attrait indéniable que représente, pour les opérateurs privés et pour les finances des pays concernés, l'exploitation de forêts "primaires" en apparence, inexploitées dans un passé récent et de ce fait riches en très gros bois d'espèces à forte valeur ajoutée?

Au mieux, lorsqu'une concession forestière est accordée à une entreprise privée pour une durée suffisamment longue, de l'ordre de vingt à trente ans, et qu'un processus d'aménagement forestier est en phase de démarrage, aucun exemple encore très probant de réelle prise en compte des pratiques coutumières et des aspirations des populations locales d'une part, de prise en considération, par des prescriptions techniques appropriées, des principales caractéristiques et des rôles écologiques des massifs forestiers concernés d'autre part, n'existe réellement et ne fait référence. II est vrai que bien des opérateurs privés se méfient fortement du poids qui pourrait être accordé dans leur concession, et ce dans le cadre des aménagements forestiers, aux questions touchant à la conservation de la diversité biologique ou à la reconnaissance des usages des populations locales. 


\section{J. VALEIX}

Dans ce domaine, les changements de comportements devront donc être recherchés, accompagnés et facilités, la viabilité de la gestion des forêts tropicales reposant bien sur une prise en compte équilibrée et pragmatique des rôles de la diversité biologique sur les processus complexes qui conditionnent la régénération naturelle, ainsi que sur une prévention des conflits fréquemment observés entre concessionnaires privés et populations locales, ces dernières étant de plus en plus soucieuses du respect, si ce n'est de la réelle prise en compte, de leurs pratiques traditionnelles.

\section{Le mythe de l'itinéraire unique}

\section{- Les limites de l'approche sylvicole}

Sur fond de reconstruction des économies suite à la Deuxième Guerre mondiale, de décolonisation, de chocs pétroliers, de croissance démographique, de mondialisation des échanges, de paupérisation de certains États du Sud, de développement du secteur privé, de désengagement des États et de déclin de l'aide publique au développement, on ne peut que prendre acte de la ténacité de la communauté forestière à viser l'objectif de la durabilité de la gestion des forêts tropicales de production essentiellement par le biais d'itinéraires techniques de type sylvicole, les réglementations forestières locales, qui confiaient un rôle généralement répressif aux services forestiers de terrain, étant pour leur part censées garantir la stabilité du foncier forestier. Ainsi, aux techniques expérimentées dès les années 1940 et 1950, telles que le "Tropical Shelterwood System" au Ghana et au Nigeria, le "Malaysian Uniform System" en Malaisie, l' "Amélioration des Peuplements naturels" en Côte-d'Ivoire, la "Sylviculture de l'Okoumé" au Congo et au Gabon, ou encore l'"Uniformisation par le haut et Normalisation" dans l'ex-Congo belge, techniques visant dans leur grande majorité une meilleure productivité des forêts tropicales grâce à une simplification à la fois de la structure des peuplements et de leur richesse spécifique, succédèrent de nombreuses expérimentations sur l'enrichissement des forêts tropicales humides, par le biais soit de reboisements en layons, soit d'éclaircies sélectives.

Inéluctablement, les aménagements de cette époque se résumèrent, outre les nécessaires travaux d'infrastructure, à une planification dans l'espace et dans le temps des opérations d'exploitation forestière et, surtout, des actions sylvicoles d'accompagnement. L'aménagement était en quelque sorte inféodé à la sylviculture, alors que, par définition, les prescriptions techniques, qu'elles soient sylvicoles ou pas, n'interviennent que dans la partie finale du processus de l'aménagement forestier, c'est-à-dire, d'une part après la première phase d'analyse et de diagnostic particulièrement capitale pour la qualité et la pertinence de l'aménagement proposé, d'autre part après la seconde phase de définition concertée et négociée des objectifs assignés à l'unité forestière à aménager.

Implicitement, les forestiers tropicalistes en charge de cette démarche cherchaient à généraliser, par changement d'échelle, les résultats des expérimentations techniques les plus probantes, conduites au préalable sur des superficies réduites et dans des conditions techniques, financières et d'encadrement tout à fait spécifiques. Cette vision partait sans doute du principe selon lequel une telle démarche relevait bien du monde de l'ingénierie forestière et la non-adoption par les acteurs locaux concernés des schémas proposés ne pouvait être imputée qu'à ces derniers et certainement pas à leurs concepteurs. Pour l'heure, le bilan de l'application de ces premières tentatives d'aménagement, assimilables plutôt à des plans de gestion, est pour le moins contrasté.

Ce bilan s'est tout d'abord avéré plutôt probant dans le contexte des grandes plantations forestières industrielles, comme en témoigne à titre d'exemple le système de gestion mis en place depuis plus d'un siècle par un groupe public indonésien dans le cadre de la gestion de 650000 hectares de teckeraie artificielle à Central Java. En zone soudano-sahélienne ensuite, plus précisément au Niger et au Mali, c'est en fait une approche intégrée de la filière bois-énergie, combinant mise en place et structuration de marchés ruraux de bois-énergie, responsabilisation 
Abattage d'un arbre émergent en forêt gabonaise

Photo J. VALEIX

des communautés locales par contractualisation avec l'administration forestière, schémas directeurs d'approvisionnement et adoption de règles fiscales incitatives, d'une part, de plans locaux de gestion sylvicole adoptant la méthode du taillis fureté, d'autre part, qui constitue, pour l'heure, l'un des exemples les plus avancés de gestion rationnelle, intégrée et, espérons-le, durable de formations arborées en zone soudano-sahélienne. En zone tropicale humide, il n'existe prati-

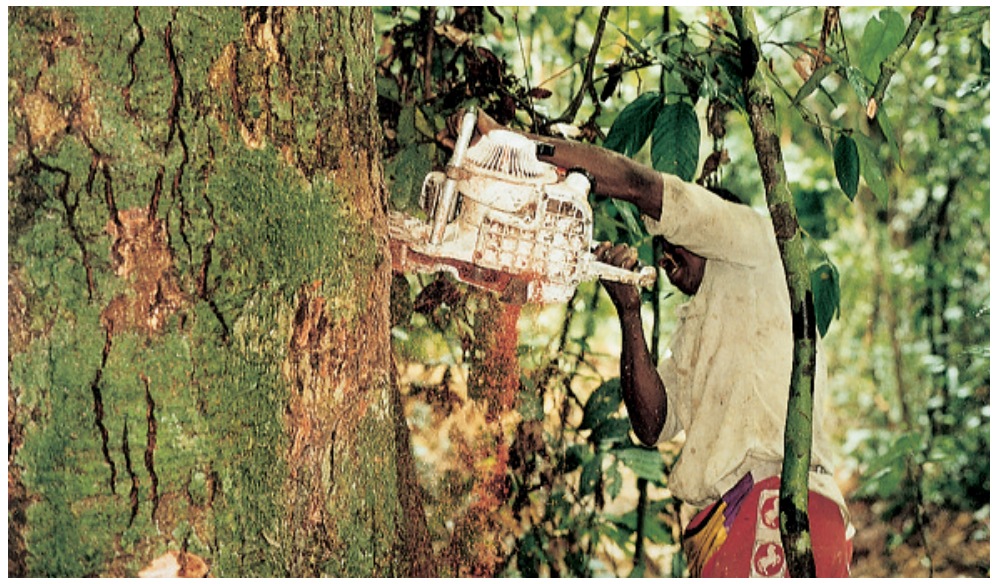
quement pas pour l'instant de projets d'aménagement de forêts naturelles, qu'elles soient "primaires" ou "secondaires", qui aient pu faire l'objet d'une mise en œuvre effective sur une durée significative, c'est-à-dire pendant au moins une dizaine d'années. Aussi, d'une manière générale, la problématique de l'aménagement des forêts tropicales humides reste-t-elle posée sur l'ensemble des continents (Afrique, Amérique, Asie...).

Citons toutefois la politique d'aménagement forestier très volontariste mise en œuvre par l'Office national des Forêts en Guyane française au cours des années 1990, ceci dans un contexte il est vrai particulier : densité de population extrêmement faible, soit 2 habitants $/ \mathrm{km}^{2}$, maîtrise du foncier par l'État, polarisation côtière et fluviale de la population guyanaise, moyens financiers du service forestier sans commune mesure avec ceux rencontrés dans les pays du Sud.

\section{- La durabilité : un concept "relatif"}

Si le concept de "gestion durable" est facile à appréhender pour des taillis ou des futaies régulières, équilibrées en classes d'âge, dont la productivité est bien connue et pour lesquels les paramètres retenus en matière de prélèvement (rotation, possibilité...) sont de nature à garantir sur le moyen et sur le long termes la durabilité de la gestion préconisée, il en va tout autrement dans le cas des forêts hétérogènes et, notamment, dans celui des forêts tropicales humides.

Ainsi, en zone tropicale humide, la première exploitation réalisée dans une forêt qualifiée jusqu'alors de "primaire" prélève un capital sur pied tout à fait atypique. Fruits de plusieurs siècles de sylvigénèse, les très gros bois alors récoltés par l'exploitant forestier ne seront pas remplacés au terme d'une rotation ni même, le plus souvent, de deux rotations, ces très gros bois correspondant en effet bien souvent à des espèces sciaphiles à croissance lente ou très lente. Les économistes forestiers parlent même en la circonstance du prélèvement d'une "rente forestière", par nature hétérogène d'une région à l'autre et même d'un écocomplexe à l'autre. Mis à part ce caractère atypique de la première exploitation en forêt "primaire", rappelons qu'il n'est pas toujours facile de disposer, pour une région forestière donnée, d'informations pertinentes et validées sur les dynamiques biologiques en jeu, notamment en ce qui concerne la croissance, les taux de mortalité et les processus de régénération propres à chaque espèce jugée principale pour l'exploitation forestière.

Par ailleurs, si la gestion des forêts tropicales doit s'exercer dans un cadre précis, celui fixé notamment par un document d'aménagement, il n'en demeure pas moins qu'elle se doit de conserver une marge de flexibilité, que ce soit par exemple en raison de l'instabilité des marchés internationaux ou locaux, de l'amélioration des connaissances sur les caractéristiques technologiques et sur les 


\section{J. VALEIX}

procédés de transformation d'espèces forestières considérées jusqu'alors comme "secondaires" ou encore de la prise en compte de telle nouvelle contrainte réglementaire édictée au niveau national. La révision anticipée d'un aménagement, voire la simple modification d'un plan de gestion, constituent à ce titre autant de voies possibles pour permettre des évolutions qui paraitraient pertinentes et souhaitables.

II semble dès lors nécessaire de démythifier la notion de gestion "durable" des forêts tropicales qui n'a réellement de sens qu'en précisant le contexte auquel elle se rapporte. Entre un objectif de maintien durable du couvert forestier, compte non tenu de la modification de sa composition floristique, et celui très théorique de maintien sur le long terme d'une possibilité-volume, sans grande variation dans le temps entre les principales espèces exploitées, se trouvent à l'évidence bien des options qui peuvent être très légitimement retenues dans le cadre du processus de l'aménagement forestier. Chercher à enrichir, à maintenir ou à réduire le capital sur pied de telle forêt tropicale ne peut en fait être analysé et évalué qu'en replaçant un tel objectif dans le contexte relatif de l'unité d'aménagement auquel il s'applique. Ainsi, tout rapport à la durabilité doit être précisé, qualifié, relativisé pour avoir un réel sens opérationnel, qui plus est en ce qui concerne la gestion des forêts tropicales. Ne parle-t-on pas d'ailleurs, de plus en plus souvent, de gestion viable des écosystèmes forestiers, un tel concept, plus relatif qu'absolu, étant par nature rattaché à un référentiel écologique, économique et social ?

\section{DES FACTEURS FAVORABLES À L'AMÉNAGEMENT DES FORÊTS TROPICALES}

\section{Un contexte international porteur}

En 1987, le rapport Brundtland de la Commission mondiale sur l'Environnement et le Développement, approuvé par l'Assemblée générale des Nations-Unies, caractérise le développement durable comme "le développement qui répond aux besoins du présent sans compromettre la capacité des générations futures à pourvoir à leurs propres besoins ».

En 1990, suite à l'objectif extrêmement ambitieux de l'Organisation internationale des Bois tropicaux (OIBT) pour l'an 2000 visant à ce que les exportations de bois tropicaux ne proviennent à cette date que de forêts aménagées, paraissent les premières directives de I'OIBT pour l'aménagement durable des forêts tropicales naturelles. Déclinées en principes et en actions possibles, elles ont pour objectif de constituer une norme de référence internationale en matière d'aménagement des forêts tropicales pour la production ligneuse, devant par la suite être complétées par des directives plus spécifiques à établir aux échelons nationaux.

Plus récemment, c'est en 1992 à Rio de Janeiro qu'est approuvé, lors des travaux de la Commission des Nations-Unies sur l'Environnement et le Développement, le programme d'action 21 selon lequel les gouvernements de la planète s'engagent à promouvoir le développement durable au niveau national ainsi que la coopération internationale. En matière forestière, cette conférence débouche, après d'âpres discussions entre pays du Nord et pays du Sud, sur une "Déclaration de principes, non juridiquement contraignante, mais faisant autorité, pour un consensus mondial sur la gestion, la conservation et l'exploitation écologiquement viable de toutes les forêts". Elle initie, surtout, la signature de trois conventions internationales majeures, relatives à la diversité biologique, aux changements climatiques et à la lutte contre la désertification.

L'année suivante, soit en 1993, paraissent simultanément les Directives de I'OIBT sur la conservation de la diversité biologique dans les forêts tropicales de production ainsi que les Directives écologiques de l'Union internationale pour la Conservation de la Nature (UICN) pour la gestion des régions forestières tropicales humides. 


\section{L'aménagement sans frontières}

II s'ensuit, en 1995, de nombreuses initiatives internationales centrées sur la définition de principes, critères et indicateurs de la gestion durable des forêts comme, par exemple :

- la proposition de Tarapoto relative à la définition de critères et indicateurs sur la durabilité des forêts amazoniennes ;

- les initiatives prises par l'Organisation africaine du Bois $(O A B)$ en matière de critères et indicateurs ;

- I'initiative prise par la FAO et le Programme des Nations-Unies pour l'Environnement (PNUE) en matière de définition de critères et d'indicateurs de gestion durable des forêts en Afrique sèche.

Outre les hétérogénéités relevées, à l'occasion de ces diverses initiatives, notamment sur les sens respectifs à accorder aux termes de "principes, critères et indicateurs" (PC\&l), d'une part, la nontransposabilité de ces PC\&I d'une région à l'autre mais aussi du niveau national au niveau local, d'autre part, il n'en demeure pas moins que les acteurs impliqués dans l'acte d'aménagement des forêts tropicales disposent désormais de nombreux documents de référence qui, bien que conçus à l'échelle internationale, au niveau régional ou dans le cadre de directives nationales, constituent autant de guides et de référentiels qu'il s'agit désormais de croiser avec l'indispensable compréhension du contexte local pour affiner le contenu des aménagements forestiers.

\section{L'évolution des contextes institutionnels nationaux et des comportements d'opérateurs privés}

Pour se mettre en phase avec leurs divers engagements internationaux ainsi qu'en réponse aux desiderata des divers bailleurs de fonds multilatéraux ou bilatéraux, de nombreux pays du Sud se dotent progressivement d'un arsenal de textes législatifs et réglementaires traitant non seulement du foncier forestier et de principes généraux s'appliquant à la gestion des espaces forestiers, mais aussi des modes d'allocation des ressources forestières et de l'aménagement forestier.

Dans de tels contextes et en s'en tenant aux seuls pays du Bassin du Congo, des opérateurs forestiers privés, généralement parmi les plus importants, s'engagent dans la démarche prônée par les administrations forestières et par les bailleurs de fonds, démarrant le plus souvent par une étude de faisabilité qui constitue une étape préalable à l'étude d'aménagement proprement dite. Par la suite, dès l'obtention d'une "concession provisoire d'aménagement-exploitation" et des moyens financiers correspondants, ils lancent l'étude d'aménagement, opération qui peut durer entre deux et trois ans compte tenu du temps que requiert tout inventaire par échantillonnage de grands massifs forestiers tropicaux.

De telles études d'aménagement sont soit sous-traitées, ce qui est encore le cas général, soit internalisées, comme cela commence à être observé au Gabon. Quelques entreprises forestières considèrent en effet que l'aménagement forestier est un processus qui ne se cantonne pas à une étude initiale, aussi poussée soit-elle, et qu'il doit faire l'objet d'une réelle mise en œuvre pouvant nécessiter la création, au sein même de l'entreprise, d'une cellule d'aménagement appelée à interagir fréquemment avec la direction et l'organisation des exploitations forestières. Ne nous y trompons pas. Si une telle évolution se confirmait et se diffusait, il s'agirait alors indiscutablement d'un changement majeur dans le comportement des opérateurs privés forestiers vis-à-vis de la gestion des forêts tropicales humides.

\section{Des forêts tropicales en quantité limitée}

$\mathrm{Si}$, pendant la majeure partie du $X X^{e}$ siècle, les forêts tropicales humides sont apparues comme inépuisables aux yeux des États du Sud et des opérateurs industriels privés, des changements importants sont venus signifier le caractère, par nature limité, des surfaces forestières exploitables. 


\section{J. VALEIX}

II y a lieu tout d'abord de rappeler la tendance régulière à l'éloignement des sites d'exploitation forestière par rapport aux côtes et donc aux ports d'exportation. Manifestation d'une tendance renforcée à la mondialisation du marché des bois tropicaux, l'installation récente de sociétés malaisiennes dans le Bassin du Congo ainsi qu'en Amérique latine n'a fait pour sa part qu'accentuer le caractère concurrentiel de l'accès aux ressources forestières. Enfin, pour les opérateurs privés ayant décidé d'investir dans un outil de transformation locale des bois tropicaux, la sécurisation de la majeure partie des approvisionnements par l'obtention de concessions de longue durée, c'est-àdire de l'ordre d'une trentaine d'années, constitue un objectif majeur qui est en grande partie conciliable avec la démarche de l'aménagement forestier, et ce pour peu que la capacité industrielle installée soit en relation avec les possibilités de production des concessions forestières attribuées à l'entreprise.

Ainsi, paradoxalement, la raréfaction des ressources forestières pourrait progressivement sonner le glas des modes de gestion traditionnels, assimilables à des exploitations minières. Ceci suppose toutefois qu'une saine concurrence entre les opérateurs privés puisse s'exercer, que des fiscalités qui incitent ces derniers à économiser les superficies des concessions comme le matériau bois soient adoptées et que, par une réglementation adaptée, les concessionnaires forestiers soient plus incités à inscrire leurs actions dans la durée, par le biais de la conception et de la mise en œuvre d'aménagements forestiers, que dans l'espace, par le biais d'une migration de leurs concessions dès le premier passage en coupe terminé.

\section{Des outils et des méthodes en progrès}

Si l'aménagement d'une forêt ne peut pas et ne doit pas se résumer à des préconisations sylvicoles, l'importance et la qualité des travaux conduits au cours de ce siècle, au sein de dispositifs expérimentaux, sur les méthodes sylvicoles applicables aux forêts tropicales ont fourni de très précieux renseignements sur la dynamique naturelle des forêts tropicales. Qu'il s'agisse de données relatives à la croissance des peuplements, à la mortalité naturelle des arbres ou encore, bien que dans une moindre mesure, à la régénération naturelle, de telles quantités d'information ont pu être intégrées dans des modèles informatiques permettant de simuler et de chercher à prédire, à moyen et à long termes, l'impact des actions anthropiques sur la dynamique des écosystèmes forestiers étudiés. De telles approches sont à l'évidence précieuses pour proposer, dans le cadre d'un processus d'aménagement, des rotations de coupes d'exploitation en phase avec la résilience de ces écosystèmes, et ceci malgré la très grande complexité écologique des formations forestières en cause.

Pour sa part, le couplage d'outils d'analyse spatiale, tels que l'imagerie satellitaire et aéroportée, d'une part, les systèmes d'information géographique et de positionnement, d'autre part, avec les méthodes d'évaluation statistique de ressources spatialisées, a déjà permis des avancées significatives dans l'estimation de ressources forestières, par nature diverses et spatialement réparties, à partir de faibles taux d'échantillonnage. Dans ce domaine toutefois, la précision de calcul des possibilités-volume qui en découlent devrait pouvoir être encore améliorée en affinant les méthodes de stratification écologique et structurale des forêts tropicales à inventorier et à aménager.

Par ailleurs, bien que reposant pour l'instant sur des approches plus ou moins empiriques, la fixation, par chaque administration forestière, de diamètres minimaux d'exploitabilité par espèce gagnerait à être confortée ou améliorée grâce aux nouvelles connaissances sur l'autécologie des principales espèces forestières, permises en partie par une utilisation accentuée des outils de biologie moléculaire. Une telle évolution s'avère, qui plus est, particulièrement souhaitable afin de minimiser les risques de surexploitation que peut engendrer la fixation de diamètres minimaux d'exploitabilité trop bas. 
Suite enfin à une intensification de l'exploitation des forêts tropicales et à un accroissement du nombre d'espèces exploitées, la rationalisation de l'organisation de l'exploitation forestière, bien connue au début du siècle compte tenu de l'importance conférée à cette époque à l'économie des moyens utilisés, est de nos jours revisitée sous le concept de l'"exploitation à faible impact". Qu'il s'agisse d'optimiser le tracé des routes et des pistes, de systématiser les inventaires d'exploitation en vue d'une exploitation plus méthodique et sans repasse ${ }^{(1)}$ des bois commercialisables, de promouvoir l'abattage directionnel, le délianage préalable et le recépage des brins d'avenir endommagés par l'exploitation, de telles lignes directrices gagneraient à l'évidence à quitter le stade de la parcelle expérimentale pour être mieux intégrées dans l'organisation courante des exploitations forestières et dans les cahiers des charges des concessions forestières.

\section{L'AMÉNAGEMENT DES FORÊTS TROPICALES À LA CROISÉE DES CHEMINS}

\section{Vers une approche holistique et intégrée}

II est souvent mentionné, dans les débats internationaux ainsi que dans les attendus des politiques forestières nationales, que l'aménagement forestier doit privilégier une approche holistique et intégrée et que les différentes fonctions de la forêt, qu'elles soient économiques, écologiques et sociales, se complètent et ne doivent pas être dissociées, même si elles peuvent faire l'objet, forêt par forêt, d'une hiérarchisation. Or, ceci s'avère nettement plus facile à dire qu'à faire. Adopter une approche holistique et intégrée présuppose en effet de très nombreuses qualités, tant professionnelles qu'humaines : grande ouverture d'esprit, aptitude au traitement et au croisement d'informations par nature très diversifiées, équilibre de la pondération attribuée aux informations écologiques, techniques, économiques et sociales, capacité de dialogue avec des acteurs aux motivations très spécifiques, pertinence de la perception des enjeux locaux...

Ne dit-on pas tout d'abord que les forêts tropicales recèlent plus de la moitié de la diversité biologique terrestre de cette planète ? Ensuite, comment traduire concrètement en règles techniques le concept relatif de "durabilité" alors que les écosystèmes forestiers tropicaux naturels sont par essence dynamiques et donc localement instables, tant dans leur composition floristique que dans leur structuration verticale ? En outre, jusqu'à quel point peut-on concilier, notamment en zone tropicale humide, la complexité des écosystèmes forestiers tropicaux, tant au plan de leur organisation spatiale que de leur fonctionnement, avec la simplicité et le caractère pragmatique attendus par les acteurs concernés, des règles de gestion préconisées dans le cadre de l'aménagement forestier? Enfin, comment intégrer dans la pratique, dans une même démarche, populations locales, administration nationale et opérateurs privés, dont les motivations sont pour le moins diverses et le plus souvent contradictoires?

En forêts tropicales humides, compte tenu de leurs très grandes diversité et complexité, l'hétérogénéité des problèmes à résoudre, d'un pays à l'autre mais aussi d'une concession forestière à l'autre, milite donc à l'évidence pour qu'un très grand soin soit apporté à la phase-clef des analyses préalables. Comprendre les enjeux locaux et nationaux, caractériser l'organisation spatiale de la diversité biologique, décrire l'organisation et l'impact sur la forêt de l'exploitation pratiquée, inventorier et effectuer la typologie des peuplements forestiers, identifier et analyser les usages forestiers des populations locales, ce n'est en définitive qu'en effectuant tout un ensemble d'analyses pertinentes et adaptées aux questions à résoudre que le processus d'aménagement forestier pourra contribuer à rendre compatibles des options jugées jusqu'alors comme antagonistes.

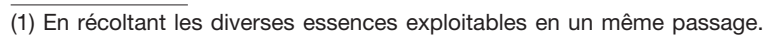




\section{J. VALEIX}

\section{Un processus de négociation, de décision et de contractualisation}

L'absence de réelle compréhension des motivations souvent contradictoires voire conflictuelles des principaux acteurs concernés par la gestion de tel espace forestier, à savoir la plupart du temps l'administration (Finances, Forêts...), les opérateurs privés et les populations locales, explique bien des échecs dans les tentatives de mise en place puis de généralisation de tel schéma de gestion ou de tel itinéraire technique. Comme l'agronomie, la foresterie ne peut échapper, surtout dans le contexte des pays en voie de développement, à une réelle compréhension ainsi qu'à une prise en compte effective du comportement et des motivations des acteurs.

S'il est généralement convenu en Europe qu'un aménagement forestier s'attache tout d'abord à l'analyse historique, technique, écologique, économique et sociale de l'unité d'aménagement, arrête ensuite les objectifs assignés à la forêt, en déduit alors les actions à réaliser et se conclut par le bilan économique et financier qui en découle, ce schéma logique ne peut être directement transposé aux foresteries du Sud. Les forêts tropicales, surtout celles situées en zone tropicale humide, sont tout d'abord bien moins connues que les forêts tempérées, à la fois en raison de leurs complexités spécifique et structurale et par manque d'archives forestières. Ensuite, malgré tous les apports possibles des phases liminaires d'acquisition de connaissances, généralement par le biais de l'imagerie spatiale, d'inventaires au sol, d'enquêtes de terrain et de recherches bibliographiques, bien des incertitudes demeureront au moment de déterminer puis de décliner aussi précisément que possible les objectifs assignés à la forêt. Enfin, fondamentalement, l'aménagement forestier constitue un processus de décision. Bien évidemment, un tel processus doit être objectivé autant que faire se peut mais il ne peut se réduire à une démarche technique, centrée sur les seules compétences, aussi grandes soient-elles, de tel ou tel aménagiste forestier.

Toutefois, si réduire l'aménagement des forêts tropicales aux seuls aspects techniques constitue une voie vouée à l'échec, la compréhension aussi fine que possible des motivations des acteurs ne suffit pas. Encore faut-il, en privilégiant les voies de la négociation et de la contractualisation, permettre que les points de vue se rapprochent et rechercher l'adoption la plus consensuelle possible d'objectifs partagés à moyen et à long termes. La communication, l'échange d'informations, la négociation et la contractualisation entre les différents acteurs doivent donc être véritablement initiés dès la première phase d'analyse pour se poursuivre au moment de la fixation des objectifs et de la détermination des diverses actions à entreprendre au sein de l'unité d'aménagement, d'une part, de la définition des contrats à passer et des cahiers des charges correspondants, d'autre part.

Une telle démarche nécessite à tout le moins une bonne maîtrise des outils et des méthodes les plus aptes à traduire pédagogiquement la complexité des écosystèmes forestiers tropicaux. Bien employés, les progrès effectués tant dans le domaine de l'imagerie spatiale, qu'elle soit aérienne ou satellitaire, dans celui des systèmes d'information, qu'ils soient à des fins de représentation cartographique ou de simulation informatique, que dans le domaine de la modélisation prédictive de la dynamique de tel type de forêt tropicale, sont de nature à faciliter la communication et le dialogue entre les différents acteurs concernés, d'une part, les prises de décision fondées sur la négociation préalable et sur une information aussi complète que possible, d'autre part.

\section{CONCLUSIONS}

Devant l'ampleur des surfaces déforestées annuellement dans les pays du Sud, il est encore trop tôt pour dire si une forte inversion de tendance dans ce domaine proviendra des bienfaits supposés d'une révolution agricole doublement verte. 


\section{L'aménagement sans frontières}

II est en tout cas certain que la prise en compte des aspirations légitimes des pays du Sud à se développer ne peut réellement faire bon ménage avec une vision trop conservatrice des forêts tropicales. À l'exception des régions les plus inhabitées et les plus éloignées, l'absence de valorisation économique des espaces forestiers constitue sans nul doute un risque majeur pour eux. Aussi, identifier dans chaque pays et pour chaque unité forestière d'aménagement les voies d'une gestion forestière viable, portée par les acteurs les plus concernés, c'est-à-dire l'État, les opérateurs privés et les populations locales, s'avère crucial, ceci d'autant plus que la valorisation des espaces forestiers doit pouvoir aller de pair avec la sauvegarde du rôle multifonctionnel des forêts tropicales.

Face aux risques déjà évoqués d'une trop grande spécialisation des espaces forestiers, que ce soit à des fins de conservation ou de production, l'un des principaux défis posés à la foresterie réside ainsi dans la mise en cohérence et dans l'intégration des fonctions économiques, écologiques et sociales des forêts tropicales. Faire cohabiter une activité d'exploitation forestière et de transformation des produits forestiers, économe, rationnelle et rentable, avec une prise en compte et une reconnaissance des usages des populations locales, notamment en ce qui concerne les prélèvements de produits forestiers non ligneux, ceci tout en préservant les caractéristiques majeures de la diversité biologique, composante essentielle de la dynamique des écosystèmes forestiers, par exemple en définissant des séries de protection mais aussi des prescriptions techniques propres à telles espèces sensibles, c'est réconcilier les usages, les rendre compatibles et se donner les moyens d'une gestion réellement viable de ces forêts.

Cela suppose toutefois, outre des actions volontaristes dans les domaines de la sensibilisation, de l'information et de la formation des acteurs, un effort réel de la part des États concernés pour définir des "règles du jeu" et des cahiers des charges qui facilitent et qui favorisent de telles évolutions.

Opter pour un choix très progressif équilibré et surtout négocié d'objectifs assignés à l'unité forestière d'aménagement, en déduire ensuite des préconisations techniques et de gestion forestière adaptées à leur contexte et pouvant être mises en œuvre par les acteurs locaux les plus directement impliqués, c'est tout d'abord rejeter le mythe de l'itinéraire unique ou d'un "prêt à penser" transposable d'un contexte à l'autre ; c'est ensuite accorder une forte priorité à la compréhension du "local", ceci en intégrant bien évidemment la dimension nationale des cadres législatifs et réglementaires ; c'est enfin resituer la démarche de l'aménagement forestier dans un processus collectif de négociation, de décision et de contractualisation qu'il n'aurait jamais dû quitter.

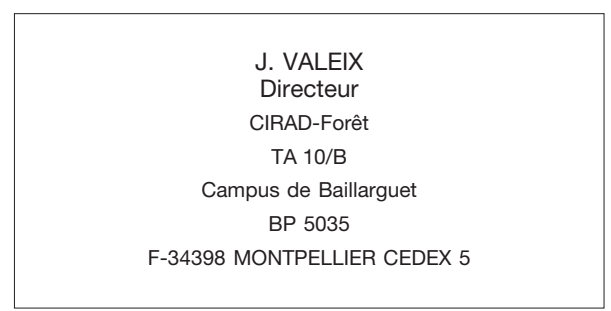

\section{BIBLIOGRAPHIE}

BERTAULT (J.-G.), KADIR (K.). - Silvicultural research in a lowland mixed dipterocarp forest of East Kalimantan. The contribution of Strek project. - Cirad-Forêt-FORDA-P.T. INHUTANI I, 1998. - 250 p.

BERTRAND (A.), BABIN (D.), NASI (R.). - Évolution et diversité du concept d'aménagement forestier. - Bois et Forêts des Tropiques, $\mathrm{n}^{\circ}$ 260, 1999, pp. 33-39.

BERTRAND (A.), BABIN (D.), NASI (R.). - L'Adaptation de l'aménagement forestier à des situations diverses. Bois et Forêts des Tropiques, $\mathrm{n}^{\circ} 261,1999$, pp. 39-48.

BERTRAND (A.), BABIN (D.), NASI (R.). - Les Composantes de l'aménagement forestier et leurs incidences financières. - Bois et Forêts des Tropiques, ${ }^{\circ} 261$, 1999, pp. 51-57. 


\section{J. VALEIX}

CATINOT (R.). - L’Aménagement durable des forêts denses tropicales humides - ATIBT. - Éditions Scytales, 1997. - $100 \mathrm{p}$.

CLÉMENT (J.). - Le Développement de la pensée et des actions forestières tropicales depuis 1946. - Bois et Forêts des Tropiques, $\mathrm{n}^{\circ}$ 252, 1997, pp. 5-24.

DUBOURDIEU (J.). - Manuel d'aménagement forestier. Gestion durable et intégrée des écosystèmes forestiers. - Paris : Office national des Forêts ; Éditions Lavoisier, 1997. - 244 p.

DURRIEU DE MADRON (L.), FORNI (E.), KARSENTY (A.), LOFFEIER (E.), PIERRE (J.-M.). - Le Projet d'aménagement pilote intégré de Dimako (Cameroun) (1992-1996). - Série FORAFRI, document $\mathrm{n}^{\circ}$ 7, $1998,160 \mathrm{p}$.

DURRIEU DE MADRON (L.), FORNI (E.), MEKOK (M.). - Les Techniques d'exploitation à faible impact en forêt dense humide camerounaise. - Série FORAFRI, document $\mathrm{n}^{\circ}$ 17, 1998, 28 p.

FAO. - Situation des forêts du monde. - Rome : Organisation des Nations-Unies pour l'Alimentation et l'Agriculture, 1999. - $156 \mathrm{p}$.

KARSENTY (A.). - Les Instruments économiques de la forêt tropicale - Le cas de l'Afrique centrale. - Éditions Maison-Neuve et Larose ; Éditions Cirad, 1999. - 125 p.

KARSENTY (A.), MENDOUGA MEBENGA (L.), PENELON (A.). - Spécialisation des espaces ou gestion intégrée des massifs forestiers. - Bois et Forêts des Tropiques, $n^{\circ} 251,1997$, pp. 43-52.

KARSENTY (A.), PIKETTY (M.G.). - Stratégies d'industrialisation fondée sur la ressource forestière et irréversibilités - Les limites de l'expérience indonésienne. - Revue Tiers-Monde, tome XXXVII, n 146, 1996, 21 p.

LOFFEIER (E.), FAVRICHON (V.). - La Forêt virtuelle. État des lieux. - Bois et Forêts des Tropiques, $\mathrm{n}^{\circ} 249$, 1996, pp. 5-19.

ORGANISATION INTERNATIONALE DES BOIS TROPICAUX (OIBT). - Directives de I'OIBT pour l'aménagement durable des forêts tropicales naturelles. - Série technique OIBT, n 5, 1990, $19 \mathrm{p}$.

ORGANISATION INTERNATIONALE DES BOIS TROPICAUX (OIBT). - Directives de I'OIBT sur la conservation de la diversité biologique dans les forêts tropicales de production. - Série OIBT : Politique forestière, $\mathrm{n}^{\circ} 5$, 1993, $20 \mathrm{p}$.

OFFICE NATIONAL DES FORÊTS (ONF). - Foresterie internationale. Textes de base et références à l'usage des forestiers francophones. - Les Dossiers forestiers, $n^{\circ}$ 4, 1999, $262 \mathrm{p}$.

PENELON (A.), MENDOUGA (L.), KARSENTY (A.). - L'Identification des finages villageois en zone forestière au Cameroun. Justification, analyse et guide méthodologique. - Série FORAFRI, document n 8, 1998,29 p.

\section{QUELLE DÉMARCHE D'AMÉNAGEMENT RETENIR EN FORÊT TROPICALE HUMIDE ? (Résumé)}

Les forêts tropicales représentent la moitié des forêts mondiales. Leur aménagement est soumis à de nombreux obstacles : la croissance démographique et des politiques volontaristes de développement économique sont des causes de déforestation ; des capacités industrielles excédentaires ont été installées en Asie, qui déplacent aujourd'hui le problème de la surexploitation vers les bassins du Congo et de l'Amazonie; la spécialisation montre ses limites aussi bien du point de vue conservationniste qui néglige les populations locales que du productivisme qui met en péril les forêts primaires; si une approche essentiellement sylvicole s'avère insuffisante, le concept de durabilité doit encore être précisé dans le cas des forêts tropicales. Cependant, des signes encourageants sont apparus avec un contexte international favorable, l'évolution des institutions nationales, celle du comportement de quelques opérateurs privés, la prise de conscience du caractère limité des ressources, les progrès de la connaissance. L'aménagement devrait ainsi évoluer vers une approche encore plus intégrée servant de support à un processus de négociation, de décision et de contractualisation.

\section{PLANNING THE MANAGEMENT OF TROPICAL RAIN FORESTS - WHAT IS THE BEST APPROACH ? (Abstract)}

Tropical forests account for half of the world's forests. Managing these forests comes up against major obstacles - population growth and active economic development policies are causes of deforestation. With surplus industrial capacities being set up in Asia, the problem of over-harvesting has moved towards the Congo and the Amazonian area. The limits of specialisation are becoming apparent in both the conservationist rationale - which neglects local populations - , and the productivist perspective - which jeopardises primary forests. Clearly, an essentially silvicultural approach is no longer adequate and the concept of sustainability as applicable to tropical forests needs to be further elaborated. Against a favourable international backdrop, some encouraging signs have appeared - changes in national institutions, in the behaviour of some private operators, an increasing awareness of the limited nature of resources and advances in knowledge. Forest management should therefore evolve towards an even more integrated approach as the foundation of a negotiating, decision-making and bargaining process. 